\title{
Existence of iterative roots for the sickle-like functions
}

Yinhe Lin ${ }^{*}$

${ }^{\text {"Correspondence: }}$ mathyhlin@163.com

Yangtze Center of Mathematics and Department of Mathematics, Sichuan University, Chengdu, Sichuan 610064, P.R. China

\begin{abstract}
The problem of iterative roots for strictly monotone self-mappings has been well solved. Most of known results concerning existence of iterative roots for a continuous function were given under the assumption that the function has finitely many non-monotonic points. When a function has infinitely many non-monotonic points, the problem of the existence of its iterative roots will become more complicated. In this paper, we study the existence of iterative roots for the sickle-like functions, as a special class of non-monotonic functions, each of which has not only one isolated non-monotonic point but also infinitely many non-isolated non-monotonic points.
\end{abstract} MSC: $37 \mathrm{E} 05 ; 39 \mathrm{~B} 12$

Keywords: non-monotonic point; sickle-like function; fixed point; iterative root

\section{Introduction}

Let $I:=[0,1]$. For any integer $n \geq 0$, consider a mapping $F: I \rightarrow I$. An iterative root of order $n$ of $F$ is a mapping $f: I \rightarrow I$ such that

$$
f^{n}(x)=F(x), \quad \forall x \in I,
$$

where $f^{n}$ denotes the $n$th iterate of the mapping $f: I \rightarrow I$, i.e. $f^{n}(x)=f\left(f^{n-1}(x)\right)$ and $f^{0}(x)=x$ for all $x \in I$ inductively. By studying the iterative roots, people can find the missing information in the iterative process. Meanwhile, being a weak version of the problem of embedding a function into a flow or into a semi-flow, the existence of iterative roots of a given mapping is a basic problem in both the theory of functional equations and the theorem of dynamical systems. The problem of finding iterative roots for a given function is still alive since the work of Babbage $[1,2]$ at the beginning of the 19th century, more and more attention has been turned to this problem (see e.g. [3-8] and references therein). Plentiful results have been obtained for continuous and strictly monotonic mappings on intervals. In the monographs $[9,10]$, Kuczma, Choczewski and Ger gave a complete description of iterative roots of continuous and strictly monotonic self-mappings on a given interval.

An interior point $x_{0}$ of $I$ is called a monotonic point of mapping $F: I \rightarrow I$ if $F$ is strictly monotonic in a neighborhood of $x_{0}$; otherwise, $x_{0}$ is referred to as a non-monotonic point or simply a fort of $F$ (see [11, 12]). Consequently, the function is strictly monotonic on $I$ if and only if it has no non-monotonic points in the interior of $I$. A function having finitely many non-monotonic points is called a strictly piecewise monotonic function or simply

@2014 Lin; licensee Springer. This is an Open Access article distributed under the terms of the Creative Commons Attribution License (http://creativecommons.org/licenses/by/2.0), which permits unrestricted use, distribution, and reproduction in any medium, provided the original work is properly cited. 
called a PM function (see [11]). Each of the non-monotonic points of a PM function is an isolated non-monotonic point. It seems that it was JZ Zhang and L Yang who first, in 1983, started to study iterative roots of PM functions explicitly in the paper [11]. They introduced the concept of characteristic interval for PM functions, and studied the existence of iterative roots of PM functions which have the characteristic interval. Later, Blokh, Coven, Misiurewicz, Nitecki and WN Zhang established some new results for iterative roots of PM functions (see $[12,13])$. Recently, there are some advances obtained for iterative roots. For example see in [14-18].

When a function has infinitely many non-monotonic points, the study of existence of its iterative roots will become more difficult. One of the typical cases that the function has infinitely many non-monotonic points is that at least there exists one nontrivial subinterval (i.e., not singleton) on which the function is constant (see e.g. [19-21]). In 1992, the author in the paper [22] proved the existence of iterative roots of a class of self-mappings possessing infinitely many non-monotonic points. Later, TX Sun and HJ Xi discussed the iterative roots of a class of self-mappings with a constant on two subintervals (see [23]).

However, in both paper [22] and paper [23], each of non-monotonic points of the function is non-isolated. The main purpose of this paper is to study the existence of continuous iterative roots for a class of functions, each of which has not only infinitely many non-isolated non-monotonic points but also one isolated non-monotonic point.

Assume that $a, b \in(0,1), a<b$ and $F \in C(I, I)$, the set of all continuous self-mappings on $I$. Then $F$ is called a sickle-like function if one of the following conditions is fulfilled: (C1) $F$ is constant on $[0, a]$, and $F$ is strictly decreasing on $[a, b]$ but strictly increasing on $[b, 1] ;(C 2) F$ is constant on $[0, a]$, and $F$ is strictly increasing on $[a, b]$ but strictly decreasing on $[b, 1]$; (C3) $F$ is constant on $[b, 1]$, and $F$ is strictly increasing on $[0, a]$ but strictly decreasing on $[a, b]$; $(C 4) F$ is constant on $[b, 1]$, and $F$ is strictly decreasing on $[0, a]$ but strictly increasing on $[a, b]$. If $F$ satisfies (C3) (resp. (C4)), then $H$ satisfies (C1) (resp. (C2)), where $H$ is defined by $H(x):=h^{-1} \circ F \circ h(x)$ for all $x \in I$, and $h: I \rightarrow I$ is defined by

$$
h(x):= \begin{cases}\frac{b-1}{a} x+1, & \forall x \in[0, a), \\ b+a-x, & \forall x \in[a, b), \\ \frac{a}{b-1}(x-1), & \forall x \in[b, 1] .\end{cases}
$$

Hence, it suffices to confine ourselves to discuss $F$ satisfying (C1) or (C2). For this purpose, let $S_{1}$ (resp. $S_{2}$ ) denote the set of all those sickle-like functions satisfying (C1) (resp. (C2)) (see Figures 1 and 2), and $S:=S_{1} \cup S_{2}$. If $F \in S$, then $b$ is an isolated non-monotonic point of $F$ but every point belonging to $[0, a]$ is a non-isolated non-monotonic point of $F$.

The paper is organized as follows: at first some important properties of iterative roots of sickle-like functions will be given in Section 2. Then in Sections 3 and 4 we will discuss the existence of iterative roots of $F \in S_{1}$ and $F \in S_{2}$, respectively. Throughout this paper, $n$ stands for a positive integer and $\left.F\right|_{E}$ represents the restriction of $F$ on $E$ for a set $E \subset I$.

\section{Preliminaries}

Lemma 2.1 Suppose that $f$ is an iterative root of order $n \geq 2$ of $F \in S$ on I. Then

(i) $f([0, a])=\{f(a)\}$ if there exists $x_{0} \in[0, a]$ such that $f\left(x_{0}\right)>a$;

(ii) $f([0, a]) \subset[0, a]$ if there exists $x_{0} \in[0, a]$ such that $f\left(x_{0}\right) \leq a$;

(iii) there exists $a^{\prime} \in[0, a)$ such that $f\left(\left[a^{\prime}, a\right]\right)=\{a\}$ if $f(a)=a$. 


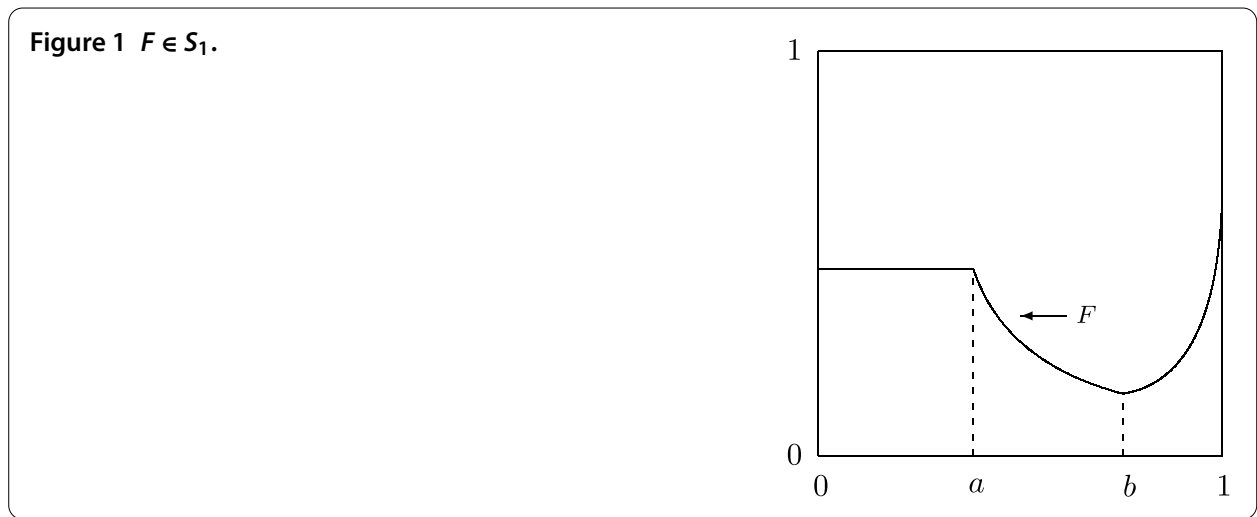

Figure $2 \quad F \in S_{2}$.

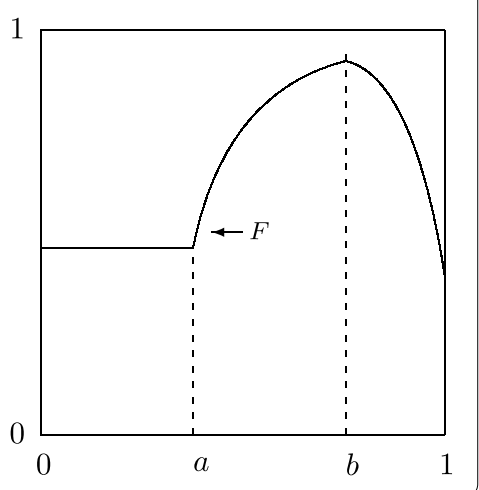

Proof For result (i), we only prove that $f([0, a])=\left\{f\left(x_{0}\right)\right\}$. By reduction to absurdity, suppose that $f([0, a]) \neq\left\{f\left(x_{0}\right)\right\}$. Then there exists $x_{*} \in[0, a]$ such that $f\left(x_{*}\right) \neq f\left(x_{0}\right)$. By the assumption, we have

$$
\begin{aligned}
& (f([0, a]) \cap[a, b]) \cup(f([0, a]) \cap[b, 1]) \\
& \quad=f([0, a]) \cap[a, 1] \supset f\left(\left[x_{0} ; x_{*}\right]\right) \cap[a, 1] \supset\left[f\left(x_{0}\right) ; f\left(x_{*}\right)\right] \cap[a, 1] \neq \emptyset,
\end{aligned}
$$

where $\left[x_{0} ; x_{*}\right]$ denotes either $\left[x_{0}, x_{*}\right]$ or $\left[x_{*}, x_{0}\right]$. It follows that at least one of $f([0, a]) \cap$ $[a, b]$ and $f([0, a]) \cap[b, 1]$ is a nonempty closed interval (not a singleton). Without loss of generality, we may assume that there are $t_{1}, t_{2} \in[a, b]$ such that $t_{1}<t_{2}$ and

$$
f([0, a]) \cap[a, b]=\left[t_{1}, t_{2}\right] .
$$

Thus, there are $t_{1}^{*}, t_{2}^{*} \in[0, a]$ such that $t_{1}^{*} \neq t_{2}^{*}, f\left(t_{1}^{*}\right)=t_{1}$ and $f\left(t_{2}^{*}\right)=t_{2}$. On the other hand, noting that $F=f \circ f^{n-1}$ is strictly monotonic on $[a, b]$, we find that $f^{n-1}$ is also strictly monotonic on $[a, b]$, which yields

$$
F\left(t_{1}^{*}\right)=f^{n}\left(t_{1}^{*}\right)=f^{n-1}\left(t_{1}\right) \neq f^{n-1}\left(t_{2}\right)=f^{n}\left(t_{2}^{*}\right)=F\left(t_{2}^{*}\right),
$$

contrary to the fact that $F$ is constant on $[0, a]$. This contradiction completes the proof of result (i).

For result (ii), we will give a proof by contradiction. Suppose that there exists $t_{3} \in[0, a)$ such that $f\left(t_{3}\right)>a$. It follows from the result (i), proved just now, that $f([0, a])=\{f(a)\}$, 
showing that $f\left(x_{0}\right)=f(a)=f\left(t_{3}\right)>a$, contrary to the assumption that $f\left(x_{0}\right) \leq a$. This contradiction shows us that $f([0, a]) \subset[0, a]$, and thus the result (ii) is proved.

For result (iii), it becomes obvious by the result (ii) that $f([0, a]) \subset[0, a]$, which shows that

$$
f^{n}([0, a])=F([0, a])=\{a\} .
$$

It follows that there exists an integer $n^{\prime} \geq 0$ such that $f^{n^{\prime}}([0, a]) \supsetneqq\{a\}$ but $f^{n^{\prime}+1}([0, a])=$ $\{a\}$. Using the continuity of $f^{n^{\prime}}$ and $f^{n^{\prime}}([0, a]) \subset[0, a]$, there exists $a^{\prime} \in[0, a)$ such that $f^{n^{\prime}}([0, a])=\left[a^{\prime}, a\right]$. Thus,

$$
f\left(\left[a^{\prime}, a\right]\right)=f^{n^{\prime}+1}([0, a])=\{a\}
$$

and the result (iii) is proved. This completes the proof of Lemma 2.1.

Lemma 2.2 Suppose that $f$ is an iterative root of order $n \geq 2$ of $F \in S$ on I. Then

(i) $f([0, a]) \subset[0, a]$ if $F(a)<a$, and

(ii) either $f([a, 1]) \subset[a, b]$ or $f([a, 1]) \subset[b, 1]$ if $F(a) \geq a$.

Proof First of all we claim that if $f(a) \geq a$, then

$$
f([a, 1]) \subset[a, 1] .
$$

Indeed, if $f(a) \geq a$, then it follows from the results (i) and (iii) of Lemma 2.1 that there exists $a^{\prime} \in[0, a)$ such that

$$
f\left(\left[a^{\prime}, a\right]\right)=\{f(a)\}
$$

Suppose that there is an $x^{\prime} \in(a, 1]$ such that $f\left(x^{\prime}\right)<a$. Let $a^{\prime \prime}:=\max \left\{a^{\prime}, f\left(x^{\prime}\right)\right\}$. Then we have

$$
\left[a^{\prime \prime}, a\right]=\left[a^{\prime}, a\right] \cap\left[f\left(x^{\prime}\right), f(a)\right] \subset\left[a^{\prime}, a\right] \cap f\left(\left[a, x^{\prime}\right]\right) .
$$

Hence, according to the continuity of $f$, there exist $s, t \in\left[a, x^{\prime}\right]$ such that $s<t$ and $f([s, t])=$ $\left[a^{\prime \prime}, a\right]$. It follows from (2.2) and (2.3) that

$$
F([s, t])=f^{n-1}(f([s, t]))=f^{n-1}\left(\left[a^{\prime \prime}, a\right]\right) \subset f^{n-1}\left(\left[a^{\prime}, a\right]\right)=\left\{f^{n-1}(a)\right\},
$$

i.e. $F$ is constant on $[s, t] \subset[a, 1]$, contradicting to the assumption on $F$. Thus the claimed (2.1) holds.

For result (i), suppose for an indirect proof that there exists $t^{*} \in[0, a]$ such that $f\left(t^{*}\right)>a$. It follows from the result (i) of Lemma 2.1 that $f([0, a])=\{f(a)\}$, which shows that $f(a)=$ $f\left(t^{*}\right)>a$. Thus (2.1) holds, implying that $F(a)=f^{n}(a) \in[a, 1]$, contradicting to the assumption and the result (i) is proved.

For result (ii), firstly we claim that

$$
f(a) \geq a .
$$


In fact, if $f(a)<a$, then there exists $\xi \in[0, a)$ such that $f(\xi)=\xi$, which implies that $F(a)=F(\xi)=f^{n}(\xi)=\xi<a$, a contradiction and thus the claimed (2.4) holds. It follows that (2.1) holds, which shows that $\left.f\right|_{[a, 1]}$ is a non-monotonic self-mapping since $\left.F\right|_{[a, 1]}$ is nonmonotonic. Note that both $\left.F\right|_{[a, b]}$ and $\left.F\right|_{[b, 1]}$ are strictly monotonic, implying that $\left.f\right|_{[a, b]}$ and $\left.f\right|_{[b, 1]}$ are both strictly monotonic. Thus, $b$ is the unique non-monotonic point of $\left.f\right|_{[a, 1]}$.

Secondly we claim that

$$
f((a, b)) \cap\{b\}=\emptyset \quad \text { and } \quad f((b, 1)) \cap\{b\}=\emptyset .
$$

Suppose, on the contrary, that $f((a, b)) \cap\{b\} \neq \emptyset$. Then there exists $t \in(a, b)$ such that $f(t)=b$. Take any neighborhood $U \subset(a, b)$ of $t$. Since $f$ is strictly monotonic in $U, f(U)$ is a neighborhood of $f(t)$, i.e. $f(U)$ is a neighborhood of $b$. Thus $f$ is not strictly monotonic in $f(U)$ as $b$ is a non-monotonic point of $f$. In other words, $f^{2}$ is not strictly monotonic in $U$. This contradicts the fact that $f^{2}$ is strictly monotonic on $[a, b]$ since $F=f^{n-2} \circ f^{2}$ is strictly increasing on $[a, b]$. This contradiction shows that the first result of (2.5) holds. Similarly, we can deduce that the second result of (2.5) holds. It follows from (2.5) that either $f([a, b]) \subset[a, b]$ or $f([a, b]) \subset[b, 1]$, and either $f([b, 1]) \subset[a, b]$ or $f([b, 1]) \subset[b, 1]$. If $f([a, b]) \subset[a, b]$, then $f([b, 1]) \subset[a, b]$. In fact, otherwise, if $f([b, 1]) \subset[b, 1]$, then $f(b)=b$. Thus $\left.f\right|_{[a, b]}$ and $\left.f\right|_{[b, 1]}$ are both strictly increasing, which together with (2.1) guarantee that $\left.f\right|_{[a, 1]}$ is a strictly increasing self-mapping. It implies that $\left.F\right|_{[a, 1]}=\left(\left.f\right|_{[a, 1]}\right)^{n}$ is also strictly increasing, a contradiction. Thus we have

$$
f([a, 1])=f([a, b]) \cup f([b, 1]) \subset[a, b] .
$$

Similarly, if $f([a, b]) \subset[b, 1]$, then $f([b, 1]) \subset[b, 1]$. It implies that

$$
f([a, 1])=f([a, b]) \cup f([b, 1]) \subset[b, 1] .
$$

Hence, the proof of Lemma 2.2 is completed.

Lemma 2.3 Suppose that $F \in S$ with $F(a) \geq a$. Then the following hold:

(i) Either $F(I) \subset[a, b]$ or $F(I) \subset[b, 1]$ if $F$ has iterative roots of order $n \geq 2$ on I;

(ii) $F$ has iterative roots of order $n \geq 2$ on I if and only if $\left.F\right|_{[a, 1]}$ has iterative roots of order $n \geq 2$ on $[a, 1]$.

Proof For result (i), suppose that $f$ is an iterative root of order $n \geq 2$ of $F$ on $I$. It follows from Lemma 2.2 that either $f([a, 1]) \subset[a, b]$ or $f([a, 1]) \subset[b, 1]$. If $f([a, 1]) \subset[a, b]$, then $f([a, b]) \subset[a, b]$, which shows, since $F([0, a])=\{F(a)\}$, that

$$
F(I)=F([a, 1])=f^{n-1}(f([a, 1])) \subset f^{n-1}([a, b]) \subset[a, b] .
$$

If $f([a, 1]) \subset[b, 1]$, then $f([b, 1]) \subset[b, 1]$. It follows that

$$
F(I)=F([a, 1])=f^{n-1}(f([a, 1])) \subset f^{n-1}([b, 1]) \subset[b, 1] .
$$

Thus the result (i) is proved. 
For result (ii), firstly we prove the sufficiency. Suppose that $f_{0}$ is an iterative root of order $n \geq 2$ of $\left.F\right|_{[a, 1]}$ on $[a, 1]$. Then, obviously, the function $f$ defined by

$$
f(x):= \begin{cases}f_{0}(a), & \forall x \in[0, a] \\ f_{0}(x), & \forall x \in(a, 1]\end{cases}
$$

is an iterative root of order $n \geq 2$ of $F$ on $I$.

Conversely, let $f$ be an iterative roots of order $n \geq 2$ of $F$ on $I$. Making use of Lemma 2.2, one obtains that $\left.f\right|_{[a, 1]}$ is a self-mapping. Thus, $\left.F\right|_{[a, 1]}=\left(\left.f\right|_{[a, 1]}\right)^{n}$, i.e. $\left.f\right|_{[a, 1]}$ is an iterative roots of order $n \geq 2$ of $\left.F\right|_{[a, 1]}$ on $[a, 1]$, and necessity is proved. This completes the proof of Lemma 2.3 .

We end this section with Lemma 2.4, which gives some basic results concerning the existence of iterative roots for strictly monotonic self-mappings.

Lemma 2.4 (see [11, Theorems 7 and 10] and [12, pp.119 and 125]) Let $m, M \in(0,1), m<M$ and $F \in C(I, I)$. Then the following statements are valid:

(i) Suppose that $F$ is strictly increasing. Then $F$ has infinitely many strictly increasing iterative roots $f$ of order $n \geq 2$ on $I$ such that $f([m, M]) \subset[F(0), F(1)]$;

(ii) Suppose that $F$ is strictly decreasing. Then $F$ has neither strictly increasing iterative roots of order $n \geq 2$ nor strictly decreasing iterative roots of even order $n \geq 2$ on I, $F$ has infinitely many strictly decreasing iterative roots of odd order $n \geq 3$ on I if and only if either $F(I) \subset(0,1)$ or $F(I)=I$. Moreover, if either $F(I) \subset(0,1)$ or $F(I)=I$, then $F$ has infinitely many strictly decreasing iterative roots $f$ of odd order $n \geq 3$ on I such that $f([m, M]) \subset[F(1), F(0)]$.

\section{Iterative roots of $F \in S_{1}$}

In this section, we discuss the existence of iterative roots of $F \in S_{1}$. It follows from Lemma 2.3 that $F$ has no iterative roots of order $n \geq 2$ if $F(a) \geq a, F(I) \not \subset[b, 1]$ and $F(I) \not \subset[a, b]$. Thus, we only consider the cases that $F(I) \subset[b, 1]$ and $F(I) \subset[a, b]$ if $F(a) \geq a$. Thus we start our discussion with Theorem 3.1.

Theorem 3.1 Suppose that $F \in S_{1}$ with $F(I) \subset[b, 1]$. Then the following hold:

(i) If either $F(1)=1$ or $F(a)<1$, then $F$ has infinitely many iterative roots of order $n \geq 2$ on I;

(ii) If $F(b)=b$ and $F(a)=1>F(1)$, then $F$ has no iterative roots of order $n \geq 2$ on I;

(iii) If $F(b)>b$ and $F(a)=1>F(1)$, then $F$ has no iterative roots of order $n \geq 3$ on I.

Proof For result (i), let $m:=F(b), M:=\max \{F(a), F(1)\}$. Then $[m, M]$ is the range of $F$ on $I$. If $F(1)<1$, then, by the assumption, we have $F(a)<1$, and thus $M<1$. If $F(1)=1$, then it is obvious that $M=1$. With the aid of Lemma 2.4 , we obtain that $\left.F\right|_{[b, 1]}$ has infinitely many strictly increasing iterative roots $f_{*}$ of order $n \geq 2$ on $[b, 1]$ such that

$$
f_{*}([m, M]) \subset[F(b), F(1)]
$$


i.e. $f_{*}$ maps $[m, M]$ into $[F(b), F(1)]$. Define the function $f: I \rightarrow I$ by

$$
f(x):= \begin{cases}\left.F\right|_{[b, 1]} ^{-1} \circ f_{*} \circ F(x), & \forall x \in[0, b), \\ f_{*}(x), & \forall x \in[b, 1] .\end{cases}
$$

Note that

$$
\left.F\right|_{[b, 1]} ^{-1} \circ f_{*} \circ F(b)=\left.\left.F\right|_{[b, 1]} ^{-1} \circ F\right|_{[b, 1]} \circ f_{*}(b)=f_{*}(b)
$$

implying that $f$ defined by (3.1) is continuous. Since

$$
\begin{aligned}
f^{n}(x) & =\left.f_{*}^{n-1} \circ F\right|_{[b, 1]} ^{-1} \circ f_{*} \circ F(x) \\
& =f_{*}^{n-1} \circ\left(f_{*}^{n}\right)^{-1} \circ f_{*} \circ F(x)=F(x), \quad \forall x \in[0, b), \\
f^{n}(x) & =f_{*}^{n}(x)=F(x), \quad \forall x \in[b, 1],
\end{aligned}
$$

we find that the function $f$ defined by (3.1) is an iterative root of order $n \geq 2$ of $F$ on $I$, and the result (i) is proved.

For result (ii), suppose, by an indirect proof, that $f$ is an iterative root of order $n \geq 2$ of $F$ on $I$. Since $F(a)>a$ and $F(I) \subset[b, 1]$, we have, on account of Lemma $2.2, f([b, 1]) \subset[b, 1]$, which shows that $\left.f\right|_{[b, 1]}$ is a strictly monotonic iterative root of order $n \geq 2$ of $\left.F\right|_{[b, 1]}$. The fact that $b=F(b)<F(1)<1$ guarantees that $\left.f\right|_{[b, 1]}$ is strictly increasing. In fact, if $\left.f\right|_{[b, 1]}$ is strictly decreasing, then at least one of $f(b)=1$ and $f(1)=b$ cannot hold. Without loss of generality, we may assume that $b \leq f(1)<f(b)<1$. Consequently,

$$
F(b)=f^{n-1}(f(b)) \in f^{n-1}([b, 1)) \subset f^{n-2}((b, 1)) \subset(b, 1) .
$$

This contradiction shows that $\left.f\right|_{[b, 1]}$ is strictly increasing. Hence, $f(b)=b$ and $f(1)<1$ since $F(b)=b$ and $F(1)<1$. Noting by Lemma 2.2 again that $f([a, 1]) \subset[b, 1]$, we infer that $f^{n-1}(a) \in[b, 1]$ which shows that

$$
F(a)=f\left(f^{n-1}(a)\right) \leq f(1)<1
$$

This contradiction completes the proof of result (ii).

For result (iii), assume by indirect proof that $f$ is an iterative root of order $n \geq 3$ of $F$ on $I$. Similarly, $\left.f\right|_{[b, 1]}$ is a strictly monotonic iterative root of order $n \geq 3$ of $\left.F\right|_{[b, 1]}$. Lemma 2.2 and the fact $F(1)<1$ force that

$$
f([a, 1]) \subset[b, 1] \text { and } f(1)<1 .
$$

If $f(b)<1$, then we deduce, by the monotonicity of $\left.f\right|_{[b, 1]}$, that

$$
f(x)<1, \quad \forall x \in[b, 1]
$$

which with (3.3) shows that (3.2) holds, a contradiction. 


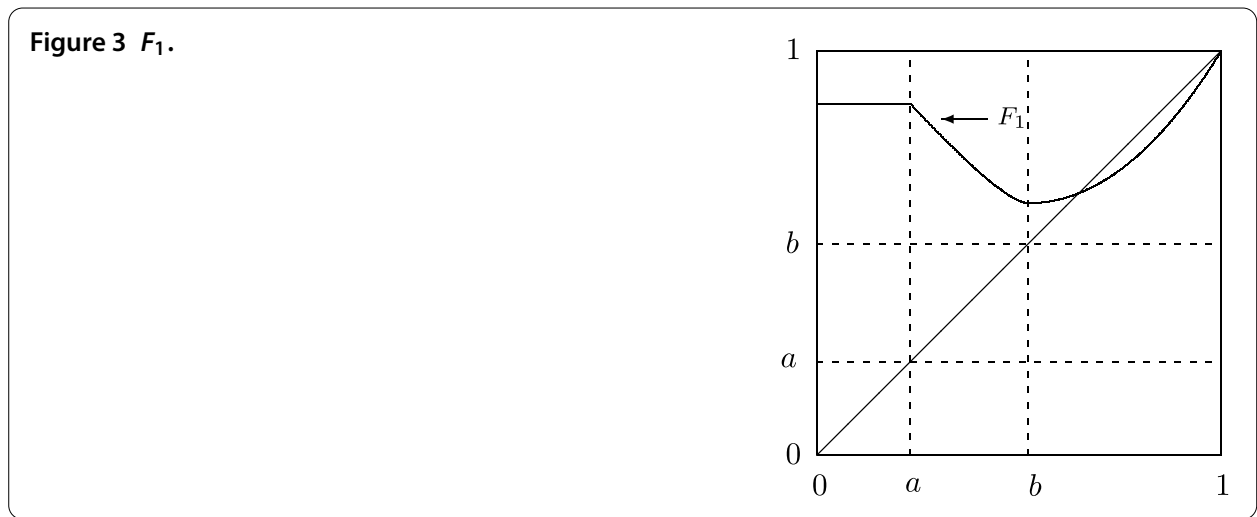

Figure $4 F_{2}$.

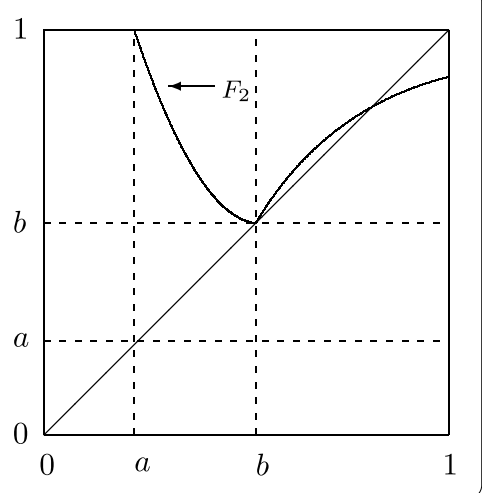

If $f(b)=1$, then $\left.f\right|_{[b, 1]}$ is strictly decreasing. Note that $F(1)<1$, implying that $f(1)>b$. It follows from (3.3) that

$$
\begin{aligned}
F(a) & =f^{n-1}(f(a)) \in f^{n-1}([b, 1])=f^{n-2}(f([b, 1])) \subset f^{n-2}((b, 1]) \\
& =f^{n-3}(f((b, 1])) \subset f^{n-3}((b, 1)) \subset(b, 1),
\end{aligned}
$$

contradicting to the assumption. Thus, the result (iii) is proved, and this completes the proof of Theorem 3.1.

Example 3.1 Consider $F_{1}: I \rightarrow I$ and $F_{2}: I \rightarrow I$ given by Figures 3 and 4, respectively.

It follows from Theorem 3.1 that $F_{1}$ has iterative roots of order $n \geq 2$ on $I$ but $F_{2}$ has no iterative roots of order $n \geq 2$ on $I$.

Theorem 3.2 Suppose that $F \in S_{1}$ with $F(I) \subset[a, b]$. Then the following statements are valid:

(i) $F$ has no iterative roots of even order $n \geq 2$ on I;

(ii) $F$ has infinitely many iterative roots of odd order $n \geq 3$ on I if and only if either $F([a, b])=[a, b]$ or $F(I) \subset(a, b)$.

Proof For result (i), suppose, for an indirect proof, that $f$ is an iterative root of even order $n \geq 2$ of $F$ on $I$. By Lemma 2.2, we see that

$$
f([a, b]) \subset f([a, 1]) \subset[a, b]
$$


which implies that $\left(\left.f\right|_{[a, b]}\right)^{n}=\left.F\right|_{[a, b]}$, i.e. $\left.f\right|_{[a, b]}$ is an iterative root of even order $n \geq 2$ of $\left.F\right|_{[a, b]}$. However, as a strictly decreasing function, we have, according to Lemma 2.4 , that $\left.F\right|_{[a, b]}$ has no iterative roots of even order. This contradiction completes the proof of result (i).

For result (ii), firstly we prove the sufficiency. Let $m:=F(b)$ and $M:=\max \{F(a), F(1)\}$. Then $[m, M]$ is the range of $F$ on $I$, and either $a=m<M=b$ or $a<m<M<b$. It follows from Lemma 2.4 that $\left.F\right|_{[a, b]}$ has infinitely many strictly decreasing iterative root $g_{*}$ of odd order $n \geq 3$ such that $g_{*}([m, M]) \subset[F(b), F(a)]$. Now we define the function $g: I \rightarrow I$ by

$$
g(x):= \begin{cases}g_{*}(x), & \forall x \in[a, b], \\ \left.F\right|_{[a, b]} ^{-1} \circ g_{*} \circ F(x), & \forall x \in I \backslash[a, b] .\end{cases}
$$

It is easy to see that $g: I \rightarrow I$ defined by (3.4) is an iterative root of odd order $n \geq 3$ of $F$ on $I$ and the sufficiency is proved.

In what follows we prove necessity. Suppose that $f$ is an iterative root of odd order $n$ of $F$ on $I$. It follows from Lemma 2.2 that

$$
f([a, b]) \subset f([a, 1]) \subset[a, b] .
$$

Thus $\left.f\right|_{[a, b]}$ is a strictly decreasing iterative root of odd order $n \geq 3$ of $\left.F\right|_{[a, b]}$. By Lemma 2.4 again, we obtain either $F([a, b])=[a, b]$ or $F([a, b]) \subset(a, b)$.

If $F([a, b]) \subset(a, b)$, which shows that at least one of $f(a)=b$ and $f(b)=a$ does not hold. Without loss of generality we may assume that $a<f(b)<f(a) \leq b$. It follows from (3.5) and the monotonicity of $\left.f\right|_{[a, b]}$ that

$$
\begin{aligned}
F(1) & =f^{n-1}(f(1)) \in f^{n-1}([a, b])=f^{n-2}(f([a, b])) \\
& \subset f^{n-2}((a, b])=f^{n-3}(f((a, b])) \subset f^{n-3}((a, b)) \subset(a, b) .
\end{aligned}
$$

Thus, $F(I)=[F(b), \max \{F(a), F(1)\}] \subset(a, b)$. The necessity is proved and the proof of Theorem 3.2 is completed.

Example 3.2 The functions $F_{3}: I \rightarrow I$ and $F_{4}: I \rightarrow I$ are given by Figures 5 and 6 , respectively.

By Theorem 3.2, we find that $F_{3}$ has exactly iterative roots of odd order $n \geq 3$ on $I$ but $F_{4}$ has no iterative roots of order $n \geq 2$ on $I$.

Figure $5 F_{3}$.

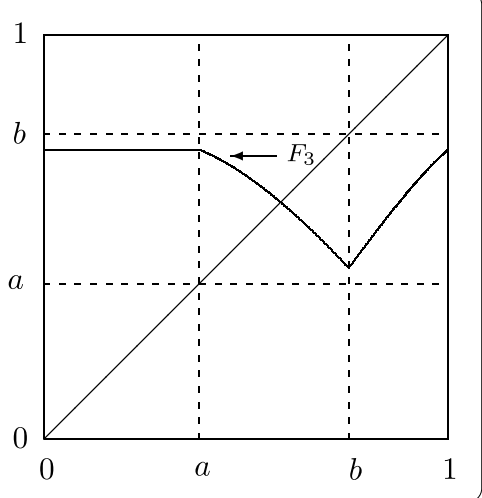




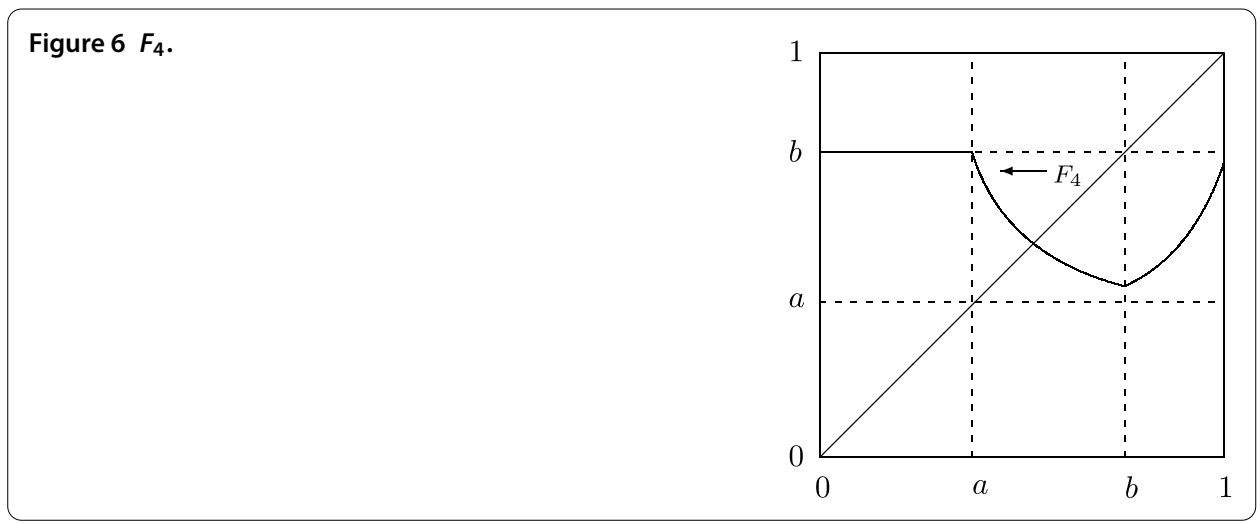

Theorem 3.3 Suppose that $F \in S_{1}$ with $F(a)<a$. Then $F$ has infinitely many iterative roots of order $n \geq 2$.

Proof Choose points $x_{1}, \ldots, x_{2 n-3}$ from $(F(a), a)$, arbitrarily, such that

$$
x_{0}:=F(a)<x_{1}<\cdots<x_{2 n-3}<x_{2 n-2}:=a .
$$

Put $I_{k}:=\left(x_{2 k-2}, x_{2 k-1}\right], I_{k}^{*}:=\left(x_{2 k-1}, x_{2 k}\right]$ for integer $k \geq 1$. Further, we define $f_{1}: I_{1} \rightarrow$ $\left[F(b), x_{0}\right)$ is a decreasing homeomorphism and $f_{1}^{*}: I_{1}^{*} \rightarrow\left(F(b), x_{0}\right]$ is an increasing homeomorphism, arbitrarily. If $n>2$, then we give the increasing homeomorphisms $f_{k}: I_{k} \rightarrow I_{k-1}$ and $f_{k}^{*}: I_{k}^{*} \rightarrow I_{k-1}^{*}$ for $k=2, \ldots, n-1$, arbitrarily. Now define the function $g_{1}$ on $[0, a]$ by

$$
g_{1}(x):= \begin{cases}x_{0}, & \forall x \in\left[0, x_{0}\right], \\ f_{k}(x), & \forall x \in I_{k}, k=1, \ldots, n-1, \\ f_{k}^{*}(x), & \forall x \in I_{k}^{*}, k=1, \ldots, n-1,\end{cases}
$$

which is continuous on $[0, a]$. Note that

$$
g_{1}^{k}(x) \in\left[F(b), x_{0}\right] \subset\left[0, x_{0}\right], \quad \forall x \in I_{k} \cup I_{k}^{*}, k=1, \ldots, n-1,
$$

implying that $g_{1}^{n}([0, a])=\left\{x_{0}\right\}$. In what follows we discuss several cases:

In the case that $F(1) \leq F(a)$ : Let the functions $g_{2}$ and $g_{3}$ be defined, respectively, by

$$
\begin{aligned}
& g_{2}(x):=f_{n-1}^{-1} \circ f_{n-2}^{-1} \circ \cdots \circ f_{1}^{-1} \circ F(x), \quad \forall x \in(a, b] \quad \text { and } \\
& g_{3}(x):=\left(f_{n-1}^{*}\right)^{-1} \circ\left(f_{n-2}^{*}\right)^{-1} \circ \cdots \circ\left(f_{1}^{*}\right)^{-1} \circ F(x), \quad \forall x \in(b, 1] .
\end{aligned}
$$

Since $f_{n-1}^{-1} \circ f_{n-2}^{-1} \circ \cdots \circ f_{1}^{-1}:\left[F(b), x_{0}\right) \rightarrow I_{n-1}$ is a decreasing homeomorphism, $\left(f_{n-1}^{*}\right)^{-1} \circ$ $\left(f_{n-2}^{*}\right)^{-1} \circ \cdots \circ\left(f_{1}^{*}\right)^{-1}:\left(F(b), x_{0}\right] \rightarrow I_{n-1}^{*}$ is an increasing homeomorphism and $F((b, 1]) \subset$ $\left(F(b), x_{0}\right]$, we find that $g_{2}:(a, b] \rightarrow I_{n-1}$ is an increasing homeomorphism, and $g_{3}:(b, 1] \rightarrow$ $I_{n-1}^{*}$ is a strictly increasing function. Moreover,

$$
g_{1}(a)=x_{2 n-4}=\lim _{x \rightarrow a+} g_{2}(x) \text { and } g_{2}(b)=x_{2 n-3}=\lim _{x \rightarrow b+} g_{3}(x)
$$


Thus the mapping $g: I \rightarrow I$ defined by

$$
g(x):= \begin{cases}g_{1}(x), & \forall x \in[0, a], \\ g_{2}(x), & \forall x \in(a, b], \\ g_{3}(x), & \forall x \in(b, 1],\end{cases}
$$

is an iterative root of order $n \geq 2$ of $F$ on $I$.

In the case that $F(1)>F(a)$ and $F$ has no fixed points on $[b, 1]$ : Define

$$
x_{2 n-1}:=b, \quad x_{2 n+k}:=\left.F\right|_{[b, 1]} ^{-1}\left(x_{k}\right)
$$

inductively for all those integer $k \geq 0$ for which the recurrence procedure (3.8) is performable. Note that $F$ has no fixed points on $[b, 1]$, implying that there is an integer $k_{0} \geq 0$ such that

$$
x_{k_{0}}<F(1) \leq x_{k_{0}+1}
$$

In fact, if $x_{k}<F(1)$ for all integers $k \geq 0$, then, by (3.6), (3.8), and the monotonicity of $\left.F\right|_{[b, 1]}$, we infer that the sequence $\left\{x_{k}\right\}$ is infinite and strictly increasing. Therefore, by (3.8), $\lim _{k \rightarrow+\infty} x_{k}(\in[b, 1])$ is a fixed point of $F$ on $[b, 1]$, a contradiction. It follows from (3.9) that $x_{2 n+k_{0}}$ exists and $x_{2 n+k_{0}}<1$. Let $x_{2 n+k_{0}+1}:=1$. Without loss of generality we may assume that $k_{0}$ is odd, and we let $k_{0}:=2 k_{0}^{\prime}+1$ for some integer $k_{0}^{\prime} \geq 0$. For $k=n, n+1, \ldots, n+k_{0}^{\prime}+1$, define $f_{k}$ on $I_{k}$ and $f_{k}^{*}$ on $I_{k}^{*}$ inductively by

$$
\begin{aligned}
& f_{k}(x):=f_{k-1}^{-1} \circ f_{k-2}^{-1} \circ \cdots \circ f_{k-(n-1)}^{-1} \circ F(x), \quad \forall x \in I_{k} \quad \text { and } \\
& f_{k}^{*}(x):=\left(f_{k-1}^{*}\right)^{-1} \circ\left(f_{k-2}^{*}\right)^{-1} \circ \cdots \circ\left(f_{k-(n-1)}^{*}\right)^{-1} \circ F(x), \quad \forall x \in I_{k}^{*},
\end{aligned}
$$

respectively. Noting that $\left.F\right|_{I_{n}}: I_{n} \rightarrow\left[F(b), x_{0}\right)$ is decreasing homeomorphism, we find that $f_{n}: I_{n} \rightarrow I_{n-1}$ is an increasing homeomorphism. Fix an $k \in\left\{n, n+1, \ldots, n+k_{0}^{\prime}\right\}$ and suppose that the function $f_{i}: I_{i} \rightarrow I_{i-1}$ is an increasing homeomorphism for $i=k, k-1, \ldots, k-n+2$. Thus $f_{k}^{-1} \circ f_{k-1}^{-1} \circ \cdots \circ f_{k-n+2}^{-1}: I_{k-n+1} \rightarrow I_{k}$ is an increasing homeomorphism. Note that $F$ : $I_{k+1} \rightarrow I_{k-n+1}$ is an increasing homeomorphism, implying by (3.10) that the function $f_{k+1}$ : $I_{k+1} \rightarrow I_{k}$ is an increasing homeomorphism. By induction, we deduce that the function $f_{k}$ : $I_{k} \rightarrow I_{k-1}$ defined by (3.10) is an increasing homeomorphism for $k=n, n+1, \ldots, n+k_{0}^{\prime}+1$. Similarly, we see that the function $f_{k}^{*}: I_{k}^{*} \rightarrow I_{k-1}^{*}$ defined by (3.11) is also an increasing homeomorphism for $k=n, n+1, \ldots, n+k_{0}^{\prime}$. Since

$$
F\left(I_{n+k_{0}^{\prime}+1}^{*}\right)=\left[F\left(x_{2 n+2 k_{0}^{\prime}+1}\right), F(1)\right] \subset\left[x_{2 k_{0}^{\prime}+1}, x_{2 k_{0}^{\prime}+2}\right]=I_{k_{0}^{\prime}+1}^{*}
$$

$f_{n+k_{0}^{\prime}+1}^{*}$ is continuous and strictly increasing on $I_{n+k_{0}^{\prime}+1}^{*}$. Define $f: I \rightarrow I$ by

$$
f(x):= \begin{cases}g_{1}(x), & \forall x \in[0, a] \\ f_{k}(x), & \forall x \in I_{k}, k=n, n+1, \ldots, n+k_{0}^{\prime}+1 \\ f_{k}^{*}(x), & \forall x \in I_{k}^{*}, k=n, n+1, \ldots, n+k_{0}^{\prime}+1\end{cases}
$$

It follows from (3.7), (3.10), and (3.11) that $f^{n}(x)=F(x)$ for all $x \in I$, i.e. the mapping $f: I \rightarrow I$, defined by (3.12), is an iterative root of order $n \geq 2$ of $F$ on $I$. 
In the case that $F(1)>F(a)$ and $F$ has fixed points on $[b, 1]:$ Let $\xi:=\min \{x: F(x)=x, x \in$ $[b, 1]\}$. Then $b<\xi$ since $F(b)<a<b$. There is no loss of generality in assuming that $\xi<1$. Because $\left.F\right|_{[\xi, 1]}$ is a strictly increasing self-mapping, and in view of Lemma $2.4,\left.F\right|_{[\xi, 1]}$ has infinitely many strictly increasing iterative roots $g_{1}^{*}$ of order $n \geq 2$ such that $g_{1}^{*}(\xi)=\xi$. Meanwhile, the infinite sequence $\left\{x_{k}\right\}$ defined by (3.6) and (3.8) is strictly increasing and $\lim _{k \rightarrow \infty} x_{k}=\xi$. Now we define a mapping $f: I \rightarrow I$ by

$$
f(x):= \begin{cases}g_{1}(x), & \forall x \in[0, a], \\ f_{k}(x), & \forall x \in I_{k}, k=n, n+1, \ldots, \\ f_{k}^{*}(x), & \forall x \in I_{k}^{*}, k=n, n+1, \ldots, \\ g_{1}^{*}(x), & \forall x \in[\xi, 1],\end{cases}
$$

where $f_{k}$ on $I_{k}$ (resp. $f_{k}^{*}$ on $I_{k}^{*}$ ) is defined inductively by (3.10) (resp. (3.11)). As we have just seen in the preceding case, it is easy to check that the mapping $f: I \rightarrow I$ defined by (3.13) is an iterative root of order $n \geq 2$ of $F$ on $I$, and the above discussion completes the proof of Theorem 3.3 .

Example 3.3 Consider the functions $F_{5}: I \rightarrow I$ and $F_{6}: I \rightarrow I$ given by Figures 7 and 8, respectively.

We know by Lemma 2.3 that $F_{5}$ has no iterative roots of order $n \geq 2$ on $I$. However, Theorem 3.3 shows that $F_{6}$ has iterative roots of order $n \geq 2$ on $I$.

Figure $7 F_{5}$.

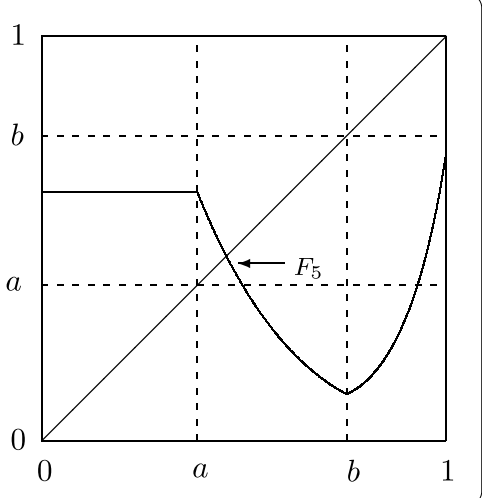

Figure $8 F_{6}$.

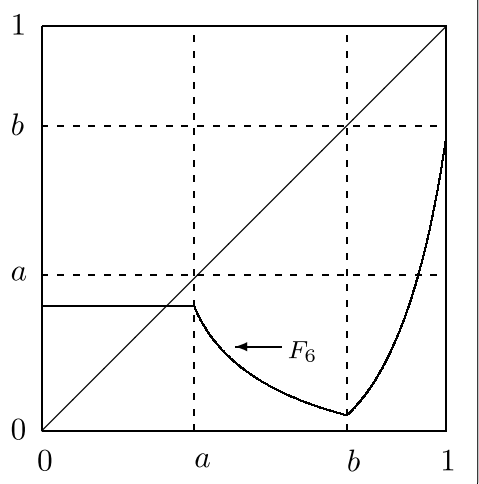




\section{Iterative roots of $F \in S_{2}$}

In this section we shall discuss the existence of iterative roots of $F \in S_{2}$. Making use of Lemma 2.3, we find that $F$ has no iterative roots of order $n \geq 2$ if $F(a) \geq a, F(I) \not \subset[b, 1]$ and $F(I) \not \subset[a, b]$. Thus, we only discuss the two cases $F(I) \subset[a, b]$ and $F(I) \subset[b, 1]$ if $F(a) \geq a$. If $F([a, 1]) \subset[a, 1]$, then, according to Lemma 2.3, it suffices to discuss the existence of iterative roots of $\left.F\right|_{[a, 1]}$. Let $G(x):=g^{-1} \circ F \circ g(x)$ for all $x \in[a, 1]$, where $g:[a, 1] \rightarrow[a, 1]$ is defined by

$$
g(x):= \begin{cases}\frac{1-b}{a-b}(x-b)+b, & \forall x \in[a, b), \\ \frac{a-b}{1-b}(x-b)+b, & \forall x \in[b, 1] .\end{cases}
$$

Thus, $G([a, 1]) \subset[a, 1]$ and $G$ is strictly decreasing on $[a, b]$ but is strictly increasing on $[b, 1]$ if $F([a, 1]) \subset[a, 1]$. By Lemma 2.3 and Theorems 3.1 and 3.2, we obtain immediately Theorems 4.1 and 4.2 .

Theorem 4.1 Suppose that $F \in S_{2}$ with $F(I) \subset[b, 1]$. Then $F$ has no iterative roots of even order $n \geq 2$ on $I$, and $F$ has infinitely many iterative roots of odd order $n \geq 3$ if and only if either $F(I) \subset(b, 1)$ or $F([b, 1])=[b, 1]$.

Theorem 4.2 Suppose that $F \in S_{2}$ with $F(I) \subset[a, b]$. Then the following statements are valid:

(i) If either $F(a)=a$ or $F(1)>a$, then $F$ has infinitely many iterative roots of order $n \geq 2$ on $I$;

(ii) If $F(b)=b$ and $F(a)>F(1)=a$, then $F$ has no iterative roots of order $n \geq 2$ on I;

(iii) If $F(b)<b$ and $F(a)>F(1)=a$, then $F$ has no iterative roots of order $n \geq 3$ on I.

Theorem 4.3 Suppose that $F \in S_{2}$ with $F(a)<a$. Then $F$ has infinitely many iterative roots of order $n \geq 2$ if and only if $F$ satisfies one of the following conditions:

(i) $F(b)<a$;

(ii) $a \leq F(b) \leq b$ and $F(a) \leq F(1)$.

Proof For sufficiency, firstly suppose that $F$ satisfies the condition (i). Choose points $a_{0}, a_{1}, \ldots, a_{3 n-2}$ from $[F(a), a]$ such that

$$
F(a)=a_{0}<F(b)=a_{1}<a_{2}<\cdots<a_{3 n-2}=a .
$$

Write $A_{k}:=\left(a_{k}, a_{k+1}\right]$ for integers $k \geq 0$. Let $\alpha_{1}: A_{1} \rightarrow A_{0}$ and $\alpha_{3}: A_{3} \rightarrow\left(F(1), a_{1}\right]$ be both arbitrary increasing homeomorphisms, and let $\alpha_{2}: A_{2} \rightarrow\left[F(1), a_{1}\right)$ be an arbitrary decreasing homeomorphism. If $n>2$, then we take increasing homeomorphisms $\alpha_{k}: A_{k} \rightarrow$ $A_{k-3}$ for $k=4,5, \ldots, 3 n-3$, arbitrarily. Define $\psi_{1}:[0, a] \rightarrow[0, a]$ by

$$
\psi_{1}(x):= \begin{cases}a_{0}, & \forall x \in\left[0, a_{1}\right], \\ \alpha_{k}(x), & \forall x \in A_{k}, k=1,2, \ldots, 3 n-3,\end{cases}
$$

which clearly is continuous on $[0, a]$ and $\psi_{1}(x) \in\left[0, a_{1}\right]$ for all $x \in\left[0, a_{4}\right]$. Thus we see that

$$
\psi_{1}^{2}(x)=a_{0}, \quad \forall x \in\left[0, a_{4}\right]
$$


Take any $x \in\left[a_{4}, a\right]$. Then there exists an $k \in\{4,5, \ldots, 3 n-3\}$ such that $x \in A_{k}$. Writing $k=3 k_{1}+k_{2}$, where $k_{1} \in\{1,2, \ldots, n-2\}$ and $k_{2} \in\{1,2,3\}$, we get

$$
\psi_{1}^{k_{1}}(x)=\alpha_{k-3\left(k_{1}-1\right)} \circ \cdots \circ \alpha_{k-3} \circ \alpha_{k}(x) \in A_{k-3 k_{1}}=A_{k_{2}} \subset\left[0, a_{4}\right] .
$$

In virtue of (4.1) and (4.2), we obtain

$$
\psi_{1}^{n}(x)=a_{0}, \quad \forall x \in[0, a]
$$

i.e. $\psi_{1}$ is an iterative root of order $n \geq 2$ of $\left.F\right|_{[0, a]}$. Define the functions $\psi_{2}$ on $(a, b]$ and $\psi_{3}$ on $(b, 1]$ by

$$
\begin{array}{ll}
\psi_{2}(x):=\alpha_{3 n-5}^{-1} \circ \cdots \alpha_{4}^{-1} \circ \alpha_{1}^{-1} \circ F(x), & \forall x \in(a, b] \quad \text { and } \\
\psi_{3}(x):=\alpha_{3 n-4}^{-1} \circ \cdots \alpha_{5}^{-1} \circ \alpha_{2}^{-1} \circ F(x), \quad \forall x \in(b, 1], &
\end{array}
$$

respectively. Note that $F((a, b])=\alpha_{1}\left(A_{1}\right)$ and $F((b, 1])=\alpha_{2}\left(A_{2}\right)$, implying that $\psi_{2}:(a, b] \rightarrow$ $A_{3 n-5}$ and $\psi_{3}:(b, 1] \rightarrow A_{3 n-4}$ are both increasing homeomorphisms. Moreover,

$$
\psi_{1}(a)=a_{3 n-5}=\lim _{x \rightarrow a+} \psi_{2}(x) \quad \text { and } \quad \psi_{2}(b)=a_{3 n-4}=\lim _{x \rightarrow b+} \psi_{3}(x)
$$

Now we define $\psi$ on $I$ by

$$
\psi(x):= \begin{cases}\psi_{1}(x), & \forall x \in[0, a], \\ \psi_{2}(x), & \forall x \in(a, b], \\ \psi_{3}(x), & \forall x \in(b, 1] .\end{cases}
$$

It is natural that

$$
\begin{array}{ll}
\psi^{n}(x)=\alpha_{1} \circ \alpha_{4} \circ \cdots \circ \alpha_{3 n-5} \circ \psi_{2}(x)=F(x), & \forall x \in(a, b] \quad \text { and } \\
\psi^{n}(x)=\alpha_{2} \circ \alpha_{5} \circ \cdots \circ \alpha_{3 n-4} \circ \psi_{3}(x)=F(x), & \forall x \in(b, 1],
\end{array}
$$

which together with (4.3) imply that the function $\psi: I \rightarrow I$ defined by (4.4) is an iterative root of order $n \geq 2$ of $F$ on $I$.

Secondly assume that $F$ satisfies the condition (ii), i.e. $a \leq F(b) \leq b$ and $F(a) \leq F(1)$. Choose points $x_{1}, \ldots, x_{n-1}$ in $(F(a), a)$, arbitrarily, such that

$$
F(a)=x_{0}<x_{1}<x_{2}<\cdots<x_{n-1}<x_{n}=a .
$$

Let $X_{k}:=\left(x_{k}, x_{k+1}\right]$ for integers $k \geq 0$. We give the increasing homeomorphisms $\beta_{k}: X_{k} \rightarrow$ $X_{k-1}$ for $k=1, \ldots, n-1$, arbitrarily. Now put

$$
x_{n+k}:=F^{-1}\left(x_{k}\right), \quad x_{n+k} \in[a, b]
$$

(which is equivalent to $x_{k}=F\left(x_{n+k}\right)$ ) for all those positive integers $k$ for which the recurrence procedure is performable. Next we shall discuss the two distinguished cases: 
In the case that $F$ has no fixed points on $[a, b]$ : Then the condition $F(a)<a$ implies that $F(b)<b$. Moreover, there exists a positive integer $k_{0}$ such that

$$
x_{k_{0}-1}<F(b) \leq x_{k_{0}} .
$$

In fact, otherwise, note that $x_{k}<F(b)$ for any integer $k \geq n$, implying, by the fact that $F(a)<a \leq x_{k}$ and (4.6), that $x_{k+1}$ exists, which shows that $x_{k}$ exists for all integers $k \geq 1$ and the sequence $\left\{x_{k}\right\}$ is strictly increasing. It follows from (4.6) that $\lim _{k \rightarrow+\infty} x_{k}$ is a fixed point of $F$ on $[a, b]$, a contradiction. The fact $x_{1}<F(b)$ yields $k_{0} \geq 2$. There is no loss of generality in assuming that $k_{0} \geq 3$. Let $x_{n+k_{0}}:=b$. Define the functions $\beta_{k}$ on $X_{k}$ inductively for $k=n, n+1, \ldots, n+k_{0}-1$ by

$$
\beta_{k}(x):=\beta_{k-1}^{-1} \circ \beta_{k-2}^{-1} \circ \cdots \circ \beta_{k-n+1}^{-1} \circ F(x), \quad \forall x \in X_{k} .
$$

Since $\left.F\right|_{X_{n}}: X_{n} \rightarrow X_{0}$ and $\beta_{n-1}^{-1} \circ \beta_{n-2}^{-1} \circ \cdots \circ \beta_{1}^{-1}: X_{0} \rightarrow X_{n-1}$ are both increasing homeomorphisms, $\beta_{n}: X_{n} \rightarrow X_{n-1}$ defined by (4.7) is an increasing homeomorphism. Similarly, by induction, we find that $\beta_{k}: X_{k} \rightarrow X_{k-1}$ defined by (4.7) is an increasing homeomorphism for $k=n, n+1, \ldots, n+k_{0}-2$. Note that $\beta_{n+k_{0}-2}^{-1} \circ \beta_{n+k_{0}-3}^{-1} \circ \cdots \circ \beta_{k_{0}}^{-1}: X_{k_{0}-1} \rightarrow X_{n+k_{0}-2}$ is an increasing homeomorphism, and we have

$$
F\left(X_{n+k_{0}-1}\right)=\left(x_{k_{0}-1}, F(b)\right] \subset\left(x_{k_{0}-1}, x_{k_{0}}\right]=X_{k_{0}-1},
$$

implying that $\beta_{n+k_{0}-1}: X_{n+k_{0}-1} \rightarrow X_{n+k_{0}-2}$ is a strictly increasing function. Define $\phi_{1}$ on $\left[x_{1}, b\right]$ by

$$
\phi_{1}(x):= \begin{cases}x_{0}, & x=x_{1}, \\ \beta_{k}(x), & \forall x \in X_{k}, k=1,2, \ldots, n+k_{0}-1\end{cases}
$$

It is natural that $\phi_{1}$ is continuous and strictly increasing on $\left[x_{1}, b\right]$. Moreover,

$$
[F(1), F(b)) \subset\left[F(a), x_{k_{0}}\right)=\left[x_{0}, x_{k_{0}}\right)=\phi_{1}\left(\left[x_{1}, x_{k_{0}+1}\right)\right) .
$$

Put $J_{0}:=[F(1), F(b))$ and define $J_{k}:=\phi_{1}^{-1}\left(J_{k-1}\right)$ inductively for $k=1,2, \ldots, n-1$. Then $J_{k} \subset$ $\left[x_{k}, x_{k+k_{0}}\right)$ for $k=0,1, \ldots, n-1$. Let the function $\phi_{2}$ on $(b, 1]$ be defined by

$$
\phi_{2}(x):=\left.\left.\left.\phi_{1}\right|_{J_{n-1}} ^{-1} \circ \phi_{1}\right|_{J_{n-2}} ^{-1} \circ \cdots \circ \phi_{1}\right|_{J_{1}} ^{-1} \circ F(x), \quad \text { for } x \in(b, 1] .
$$

Because $F:(b, 1] \rightarrow J_{0}$ is a decreasing homeomorphism and $\left.\phi_{1}\right|_{J_{k}} ^{-1}: J_{k-1} \rightarrow J_{k}$ is an increasing homeomorphism for $k=1,2, \ldots, n-1$, we infer that $\phi_{2}:(b, 1] \rightarrow J_{n-1}$ is a decreasing homeomorphism. By the definition of $\phi_{1}$, we possess that $\left.\phi_{1}\right|_{X_{k}} ^{-1}\left(=\beta_{k}^{-1}\right): X_{k-1} \rightarrow X_{k}$ is an increasing homeomorphism for $k=1,2, \ldots, n+k_{0}-1$. On the other hand, noting that $F(b) \in\left(x_{k_{0}-1}, x_{k_{0}}\right]$ and $\left.F\right|_{[b, 1]}$ is strictly decreasing, we have $b_{*} \in(b, 1)$ such that

$$
F\left(\left(b, b_{*}\right)\right)=\left(F\left(b_{*}\right), F(b)\right) \subset J_{0} \cap\left(x_{k_{0}-1}, x_{k_{0}}\right) \subset J_{0} \cap X_{k_{0}-1} .
$$


Thus

$$
\begin{aligned}
\phi_{2}(x) & :=\left.\left.\left.\phi_{1}\right|_{J_{n-1}} ^{-1} \circ \phi_{1}\right|_{J_{n-2}} ^{-1} \circ \cdots \circ \phi_{1}\right|_{J_{1}} ^{-1} \circ F(x) \\
& =\left.\left.\left.\phi_{1}\right|_{X_{n+k_{0}-2}} ^{-1} \circ \phi_{1}\right|_{X_{n+k_{0}-3}} ^{-1} \circ \cdots \circ \phi_{1}\right|_{X_{k_{0}}} ^{-1} \circ F(x) \\
& =\beta_{n+k_{0}-2}^{-1} \circ \beta_{n+k_{0}-3}^{-1} \circ \cdots \circ \beta_{k_{0}}^{-1} \circ F(x), \quad \forall x \in\left(b, b_{*}\right) .
\end{aligned}
$$

This shows that

$$
\lim _{x \rightarrow b+} \phi_{2}(x)=\beta_{n+k_{0}-2}^{-1} \circ \beta_{n+k_{0}-3}^{-1} \circ \cdots \circ \beta_{k_{0}}^{-1} \circ F(b)=\phi_{1}(b) .
$$

Define $\phi: I \rightarrow I$ by

$$
\phi(x):= \begin{cases}x_{0}, & \forall x \in\left[0, x_{1}\right), \\ \phi_{1}(x), & \forall x \in\left[x_{1}, b\right] \\ \phi_{2}(x), & \forall x \in(b, 1]\end{cases}
$$

which is continuous since $\lim _{x \rightarrow x_{1}-} \phi(x)=x_{0}=\phi_{1}\left(x_{1}\right)$ and (4.8). By the definition of $\phi_{1}$ and $\phi_{2}$, we see that $\phi: I \rightarrow I$ defined by (4.9) is an iterative root of order $n \geq 2$ of $F$ on $I$.

In the case that $F$ has fixed points on $[a, b]:$ Let $\xi=\min \{x: F(x)=x, x \in[a, b]\}$. Then $x_{k}$ is well defined inductively by (4.6) for all integers $k \geq n$ and $\lim _{k \rightarrow \infty} x_{k}=\xi$. Without loss of generality, assume that $\xi<b$. Let $\beta_{k}: X_{k} \rightarrow X_{k+1}$ be defined by (4.7) for all integers $k \geq n$. If $x \in(a, \xi)$, then there exists $X_{k}$ such that $x \in X_{k}$, which implies

$$
x_{k-1}=\beta_{k}\left(x_{k}\right)<\beta_{k}(x) \leq \beta_{k}\left(x_{k+1}\right)=x_{k} .
$$

Noting that $x \rightarrow \xi$ is equivalent to $k \rightarrow \infty$, we get the conclusion that

$$
\lim _{k \rightarrow \infty} x_{k-1} \leq \lim _{x \rightarrow \xi-0} \beta_{k}(x) \leq \lim _{k \rightarrow \infty} x_{k}
$$

Hence,

$$
\lim _{x \rightarrow \xi-0} \beta_{k}(x)=\xi
$$

On the other hand, since $\left.F\right|_{[\xi, b]}$ is a strictly increasing self-mapping and $F(\xi)=\xi$, we obtain, in view of Lemma 2.4 , that $\left.F\right|_{[\xi, b]}$ has infinitely many strictly increasing iterative roots $\beta_{*}$ of order $n \geq 2$ on $[\xi, b]$ such that

$$
\beta_{*}(\xi)=\xi
$$

Define the function $\phi_{3}$ on $\left(x_{1}, b\right]$ by

$$
\phi_{3}(x):= \begin{cases}\beta_{k}(x), & \forall x \in X_{k}, k=1,2, \ldots \\ \beta_{*}(x), & \forall x \in[\xi, b] .\end{cases}
$$

By the definition of $\beta_{k}$ and $\beta_{*},(4.10)$ and (4.11), we see that the function $\phi_{3}$ on $\left(x_{1}, b\right]$ defined by (4.12) is continuous and strictly increasing. Let $\mathcal{J}_{0}:=[F(1), F(b)) . \mathcal{J}_{k}:=\phi_{3}^{-1}\left(\mathcal{J}_{k-1}\right)$ 
is well defined inductively for $k=1,2, \ldots, n-1$. Thus $\left.\phi_{3}\right|_{\mathcal{J}_{k}} ^{-1}: \mathcal{J}_{k-1} \rightarrow \mathcal{J}_{k}$ is an increasing homeomorphism for $k=1,2, \ldots, n-1$. Then the function $\phi_{4}$ on $(b, 1]$ given by

$$
\phi_{4}(x):=\left.\left.\left.\phi_{3}\right|_{\mathcal{J}_{n-1}} ^{-1} \circ \phi_{3}\right|_{\mathcal{J}_{n-2}} ^{-1} \circ \cdots \circ \phi_{3}\right|_{\mathcal{J}_{1}} ^{-1} \circ F(x), \quad \forall x \in(b, 1],
$$

is a decreasing homeomorphism from $(b, 1]$ to $\mathcal{J}_{n-1}$. By the definition of $\phi_{3}$ and $\beta_{*}$, we deduce that, for $k=1,2, \ldots, n-1$,

$$
\left.\phi_{3}\right|_{\mathcal{J}_{k}} ^{-1}(y)=\left.\phi_{3}\right|_{(\xi, b)} ^{-1}(y)=\beta_{*}^{-1}(y), \quad \forall y \in \beta_{*}((\xi, b)) \cap \mathcal{J}_{k-1} .
$$

Choose $b^{*} \in(b, 1)$ satisfying

$$
F\left(\left(b, b^{*}\right)\right) \subset(\xi, F(b)) \cap \mathcal{J}_{0}=\beta_{*}^{n}((\xi, b)) \cap \mathcal{J}_{0} .
$$

Consequently, for $k=1,2, \ldots, n-1$,

$$
\begin{aligned}
\beta_{*}^{-k+1} \circ F(x) & =\phi_{3}^{-k+1} \circ F(x) \in \phi_{3}^{-k+1}\left((\xi, F(b)) \cap \mathcal{J}_{0}\right) \\
& \subset \beta_{*}^{n-k+1}((\xi, b)) \cap \mathcal{J}_{k-1} \subset \beta_{*}((\xi, b)) \cap \mathcal{J}_{k-1}, \quad \forall x \in\left(b, b^{*}\right) .
\end{aligned}
$$

It follows from (4.14) that

$$
\left.\phi_{3}\right|_{\mathcal{J}_{k}} ^{-1}\left(\beta_{*}^{-k+1} \circ F(x)\right)=\beta_{*}^{-k} \circ F(x), \quad \forall x \in\left(b, b^{*}\right), k=1,2, \ldots, n-1,
$$

which jointly with (4.13) guarantees that

$$
\phi_{4}(x)=\beta_{*}^{-n+1} \circ F(x), \quad \forall x \in\left(b, b^{*}\right),
$$

showing that

$$
\lim _{x \rightarrow b+} \phi_{4}(x)=\lim _{x \rightarrow b+} \beta_{*}^{-n+1} \circ F(x)=\beta_{*}^{-n+1} \circ F(b)=\beta_{*}(b)=\phi_{3}(b) .
$$

In addition, $\lim _{x \rightarrow x_{1}+} \phi_{3}(x)=x_{0}$. Consequently, the mapping $\tilde{\phi}: I \rightarrow I$ defined by

$$
\tilde{\phi}(x):= \begin{cases}x_{0}, & \forall x \in\left[0, x_{1}\right], \\ \phi_{3}(x), & \forall x \in\left(x_{1}, b\right], \\ \phi_{4}(x), & \forall x \in(b, 1],\end{cases}
$$

is continuous. According to (4.12) and (4.13), the mapping $\tilde{\phi}: I \rightarrow I$ defined by (4.15) is an iterative root of order $n \geq 2$ of $F$ on $I$. Consequently, the sufficiency is proved.

To prove necessity, suppose that $f$ is an iterative root of order $n \geq 2$ of $F$ on $I$. It follows from Lemma 2.2 that

$$
f([0, a]) \subset[0, a]
$$

which implies that $f$ has the fixed points on $[0, a]$. Note that the fixed point of $f$ is bound to the fixed point of $F$ and $F(a)$ is the unique fixed point of $F$ on $[0, a]$, implying that $F(a)$ 
is the unique fixed point of $f$ on $[0, a]$. It follows that

$$
f(a)<a, \quad f(F(a))=F(a) .
$$

Since $f^{n}([0, a])=F([0, a])=\{F(a)\}$ and $f^{0}([0, a])=[0, a]$, there exists $t_{0} \in\{0,1, \ldots, n-1\}$ such that $f^{t_{0}}([0, a]) \neq\{F(a)\}$ but $f^{t_{0}+1}([0, a])=\{F(a)\}$. By the continuity of $f^{t_{0}}$, there are $p, q \in[0, a]$ such that $p<q$ and $[p, q]=f^{t_{0}}([0, a])$. Thus the definition of $t_{0}$ and (4.17) yields

$$
F(a) \in[p, q] \text { and } f(x)=F(a), \quad \forall x \in[p, q] .
$$

We claim that

$$
f([0, a]) \cap f((a, b])=\emptyset .
$$

Otherwise, if there exist $x^{\prime} \in[0, a]$ and $x^{\prime \prime} \in(a, b]$ such that $f\left(x^{\prime}\right)=f\left(x^{\prime \prime}\right)$, then $F\left(x^{\prime}\right)=$ $f^{n}\left(x^{\prime}\right)=f^{n}\left(x^{\prime \prime}\right)=F\left(x^{\prime \prime}\right)$, which contradicts to the fact that $F\left(x^{\prime}\right)=F(a)<F\left(x^{\prime \prime}\right)$. Therefore, as claimed (4.19) holds. On the other hand, $\left.f\right|_{[a, b]}$ and $\left.f\right|_{[b, 1]}$ are both strictly monotonic since $\left.F\right|_{[a, b]}$ and $\left.F\right|_{[b, 1]}$ are both strictly monotonic. In what follows we shall distinguish the following several cases:

In the case that $f(a)<F(a)$ : Suppose for indirect proof that $F(b) \geq a$. Then there exists $b^{\prime} \in(a, b]$ such that $F\left(b^{\prime}\right)=a$ since $F(a)<a$. On the other hand, we have, in virtue of (4.17) and (4.19),

$$
[f(a), F(a)] \cap f((a, b]) \subset f([F(a), a]) \cap f((a, b]) \subset f([0, a]) \cap f((a, b])=\emptyset .
$$

It follows that $\left.f\right|_{[a, b]}$ is strictly decreasing. Thus

$$
f(a)>f(x), \quad \forall x \in(a, b] .
$$

By (4.17) and (4.20), we get $f\left(b^{\prime}\right)<f(a)<a$. Thus

$$
f(a)=f\left(F\left(b^{\prime}\right)\right)=f\left(f^{n}\left(b^{\prime}\right)\right)=f^{n}\left(f\left(b^{\prime}\right)\right)=F\left(f\left(b^{\prime}\right)\right)=F(a),
$$

contradicting to the assumption that $f(a)<F(a)$. This contradiction shows that $F(b)<a$ holds, i.e. $F$ satisfies the condition (i).

In the case that $f(a)=F(a)$ : Firstly we prove that $\left.f\right|_{[a, b]}$ is strictly decreasing. In fact, if $\left.f\right|_{[a, b]}$ is strictly increasing, then, by (4.19), we infer that

$$
f([0, a]) \cap(f(a), f(b)]=f([0, a]) \cap f((a, b])=\emptyset .
$$

This forces $f(x)$ to obey

$$
f(x) \leq f(a), \quad \forall x \in[0, a]
$$

which with (4.16) implies that

$$
f^{n-1}(x)=f\left(f^{n-2}(x)\right) \leq f(a), \quad \forall x \in[0, a] .
$$


Note that $(f(a), f(b)] \cap(f(a), a] \neq \emptyset$, implying that there exists $t^{\prime} \in(a, b]$ such that $f\left(t^{\prime}\right) \leq a$, which with (4.22) results in

$$
F\left(t^{\prime}\right)=f^{n-1}\left(f\left(t^{\prime}\right)\right) \leq f(a)=F(a)
$$

contradicting to the fact that $F\left(t^{\prime}\right)>F(a)$. Thus, $\left.f\right|_{[a, b]}$ is strictly decreasing. It follows from (4.17) that

$$
f(b)<f(a)<a,
$$

which with (4.16) means that

$$
F(b)=f^{n}(b) \leq a
$$

Based on (4.19), we have

$$
f([0, a]) \cap[f(b), f(a))=f([0, a]) \cap f((a, b])=\emptyset,
$$

which with (4.16) shows that

$$
f([0, a]) \subset[f(a), a]
$$

Moreover, we have

$$
f(1) \leq a
$$

In fact, if $f(1)>a$, then, by (4.23), $\left.f\right|_{[b, 1]}$ is strictly increasing. Note that $F(a)=f(a) \in$ $(f(b), f(1))$, implying by $(4.18)$ that $[p, q] \cap(f(b), f(1)) \neq \emptyset$. Thus there exist $s, t \in(b, 1)$ such that $s<t$ and $f([s, t])=[p, q] \cap(f(b), f(1))$. It follows thus from (4.18) that

$$
F([s, t])=f^{n}([s, t])=f^{n-1} \circ f([s, t]) \subset f^{n-1}([p, q])=\left\{f^{n-2}(F(a))\right\}
$$

i.e. $F$ is constant on $[s, t]$. This contradiction shows that the claimed (4.26) holds. Making use of (4.25) and (4.26), one obtains

$$
F(1)=f^{n}(1)=f^{n-1}(f(1)) \in f^{n-1}([0, a]) \subset f^{n-2}([f(a), a]) \subset[f(a), a]=[F(a), a],
$$

which with (4.24) guarantees that $F$ satisfies one of conditions (i) and (ii).

In the case that $f(a)>F(a)$ : It is natural by (4.17) and (4.19) that

$$
[F(a), f(a)] \cap f((a, b]) \subset f([0, a]) \cap f((a, b])=\emptyset .
$$

Thus $\left.f\right|_{[a, b]}$ is strictly increasing. Due to (4.19), we have $f([0, a]) \cap(f(a), f(b)]=\emptyset$. It follows that (4.21) holds.

If $f(b) \leq a$, then (4.16) and (4.21) imply that $F(b)=f\left(f^{n-1}(b)\right) \leq f(a)<a$, i.e., $F$ satisfies the condition (i). 
If $f(b)>a$, by the continuity and monotonicity of $\left.f\right|_{[a, b]}$ and (4.17), there is an $a_{-1} \in(a, b)$ such that $f\left(a_{-1}\right)=a$. Let $a_{k}:=f\left(a_{k-1}\right)$ be well defined inductively for $k=0,1, \ldots, n$. It follows thus from (4.17) that $a_{1}<a$. Since $\left.F\right|_{\left[a, a_{-1}\right]}\left(=\left.\left.f^{n-2} \circ f\right|_{\left[a_{1}, a\right]} \circ f\right|_{\left[a, a_{-1}\right]}\right)$ is strictly increasing, we see that $\left.f\right|_{\left[a_{1}, a\right]}$ is strictly monotonic, which together with (4.21) implies that $\left.f\right|_{\left[a_{1}, a\right]}$ is strictly increasing. Fix a $k \in\{0,1, \ldots, n-2\}$, arbitrarily. Assume that $\left.f\right|_{\left[a_{i}, a_{i-1}\right]}$ is strictly increasing for each $i \in\{0,1,2, \ldots, k\}$. Noting that $f\left(\left(a_{-1}, b\right)\right) \cap\left(a, a_{-1}\right] \neq \emptyset$, there is an $a_{*} \in$ $\left(a_{-1}, b\right)$ such that $f\left(a_{*}\right) \in\left(a, a_{-1}\right]$. Whence, by the induction hypothesis, we infer that

$$
\left.\left.\left.\left.f\right|_{\left[a_{k}, a_{k-1}\right]} \circ f\right|_{\left[a_{k-1}, a_{k-2}\right]} \circ \cdots \circ f\right|_{\left[a, a_{-1}\right]} \circ f\right|_{\left[a_{-1}, a_{*}\right]}
$$

is strictly increasing, i.e. $\left.f^{k+2}\right|_{\left[a_{-1}, a_{*}\right]}$ is strictly increasing. Because $\left.F\right|_{\left[a, a_{*}\right]}\left(=f^{n-k-2}\right.$ 。 $\left.\left.f^{k+2}\right|_{\left[a, a_{*}\right]}\right)$ is strictly increasing, we see that $\left.f^{k+2}\right|_{\left[a, a_{*}\right]}$ is strictly monotonic. It follows that $\left.f^{k+2}\right|_{\left[a, a_{-1}\right]}$ is strictly increasing. Thus, by the induction hypothesis,

$$
\left.\left.\left.\left.f\right|_{\left[a_{k+1}, a_{k}\right]} \circ f\right|_{\left[a_{k}, a_{k-1}\right]} \circ \cdots \circ f\right|_{\left[a_{1}, a\right]} \circ f\right|_{\left[a, a_{-1}\right]}
$$

is strictly increasing, which shows that $\left.f\right|_{\left[a_{k+1}, a_{k}\right]}$ is strictly increasing. Consequently, by induction, $\left.f\right|_{\left[a_{k}, a_{k-1}\right]}$ is strictly increasing for $k=0,1, \ldots, n-1$. Thus

$$
F(a)=a_{n}<a_{n-1}<\cdots<a_{2}<a_{1}<a .
$$

Moreover, $\left.f\right|_{\left[a_{n-1}, b\right]}$ is strictly increasing, showing that

$$
f(x)>F(a), \quad \forall x \in\left(a_{n-1}, b\right] .
$$

Meanwhile, it can be seen that

$$
f\left(\left[a_{n}, a_{n-1}\right]\right)=f \circ f\left(\left[a_{n-1}, a_{n-2}\right]\right)=\cdots=f^{n}\left(\left[a_{1}, a\right]\right)=F\left(\left[a_{1}, a\right]\right)=\{F(a)\} .
$$

If $\left.f\right|_{[b, 1]}$ is strictly increasing, then it follows from (4.27) and (4.28) that $\left.f\right|_{[F(a), 1]}$ is an increasing self-mapping, which forces that $\left.F\right|_{[F(a), 1]}$ is an increasing self-mapping. This contradiction shows that $\left.f\right|_{[b, 1]}$ must be strictly decreasing. Thus the point $b$ is a nonmonotonic point of $f$. If $f(b)>b$, then there exists $b_{1} \in(a, b)$ such that $f\left(b_{1}\right)=b$. Consequently, $b_{1}$ is a non-monotonic point of $f^{2}$. However, $f^{2}$ is strictly monotonic on $[a, b]$ since $\left.f^{n-2} \circ f^{2}\right|_{[a, b]}\left(=\left.F\right|_{[a, b]}\right)$ is strictly increasing. This contradiction yields

$$
f(b) \leq b \text {. }
$$

Further, we claim that

$$
f(1) \geq F(a)
$$

In fact, if $f(1)<F(a)$, then, by (4.27), $F(a) \in(f(1), f(b))$ and (4.18) shows that $[p, q] \cap$ $(f(1), f(b)) \neq \emptyset$. As the proof of (4.26), we can obtain a contradiction. It follows from (4.27), (4.28), (4.29), and (4.30) that $\left.f\right|_{[F(a), 1]}$ is a self-mapping, and we have

$$
f(x) \leq b, \quad \forall x \in[F(a), 1]
$$




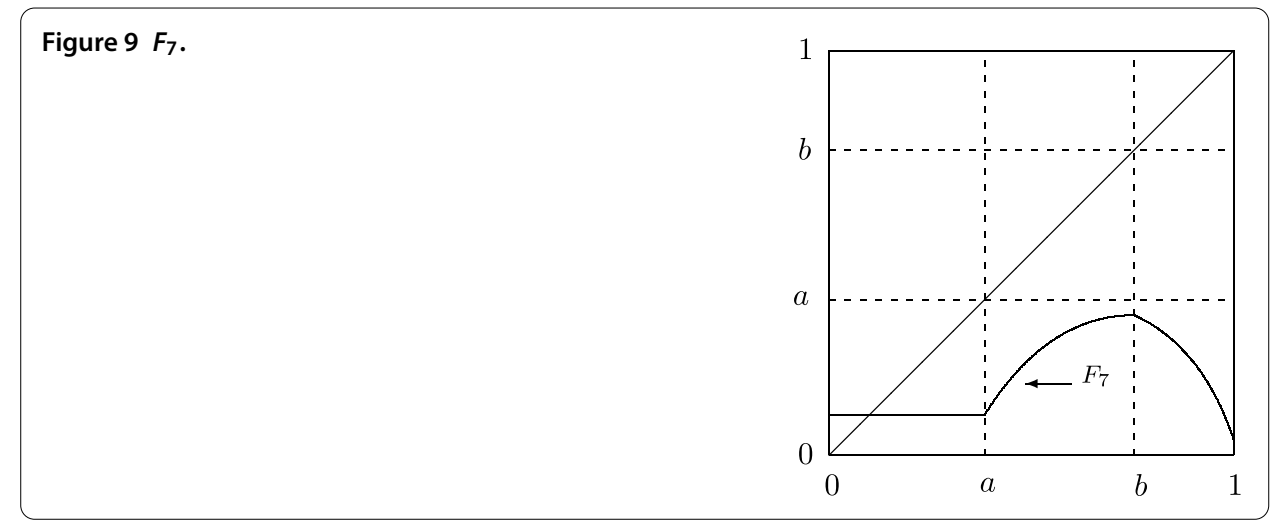

Figure $10 F_{8}$.

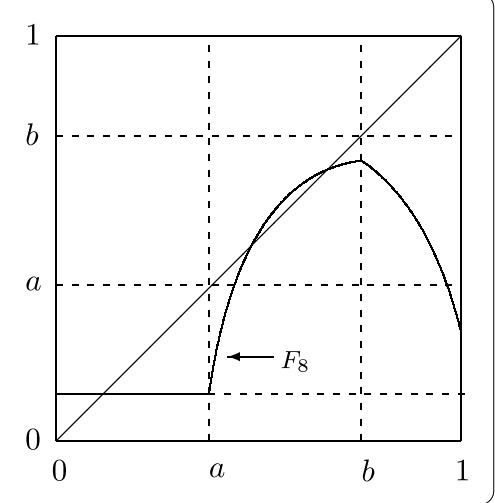

Figure $11 F_{9}$.

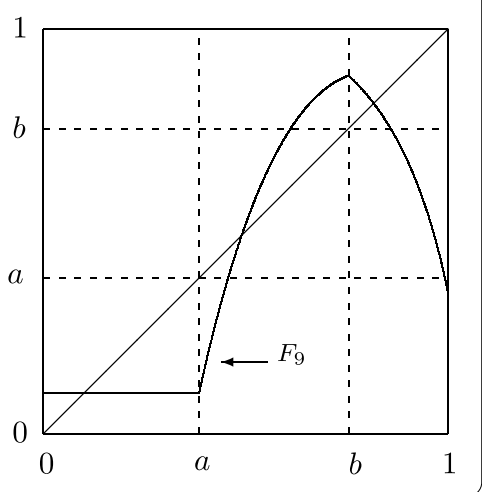

which implies that $F(b)=f\left(f^{n-1}(b)\right) \leq b$ and $F(1)=f^{n}(1) \geq F(a)$. Consequently, $F$ satisfies the condition (ii) if $F$ does not satisfy the condition (i), and necessity is proved. This completes the proof.

Example 4.1 Let $F_{7}: I \rightarrow I$ and $F_{8}: I \rightarrow I$ be given by Figures 9 and 10, respectively.

We see, according to Theorem 4.3 , that both $F_{7}$ and $F_{8}$ have iterative roots of order $n \geq 2$ on $I$.

Example 4.2 It follows from Theorem 4.3 that the functions $F_{9}: I \rightarrow I$ and $F_{10}: I \rightarrow I$ given by Figures 11 and 12, respectively, have no iterative roots of order $n \geq 2$ on $I$. 


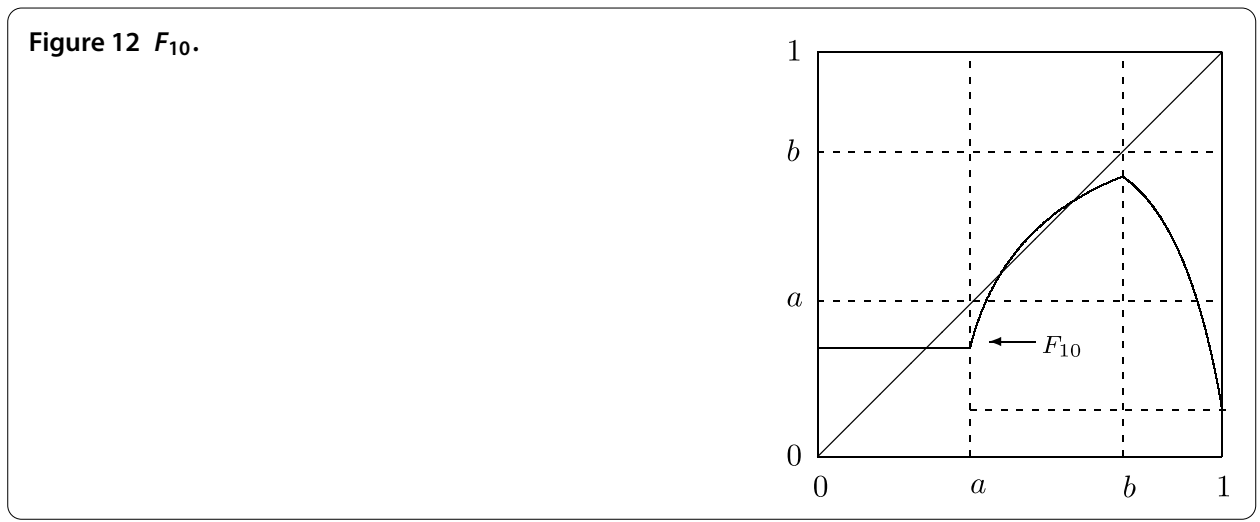

In this paper, we give all conditions of existence and nonexistence of iterative roots for the sickle-like functions. Meanwhile, we construct infinite many iterative roots if they exist. However, we are not sure if all continuous iterative roots are defined by the construction method given in this paper, in other words, it is unclear whether there are other forms of iterative roots if they exist. On the other hand, as the number of the subintervals on which the function is constant and isolated non-monotonic points increases, we do not know whether the existence of its iterative roots can be solved in a similar manner to the one described in this paper. Although we are not able to give answers for these problems, yet, they are our further directions of investigation.

\section{Competing interests}

The author declares that he has no competing interests.

Author's contributions

The author read and approved the final manuscript.

\section{Acknowledgements}

The author would like to express sincere gratitude to the editors and the referees for being kind enough to give very helpful suggestions and make many comments. This research was supported by the Grant No. LY14A010005 of Zhejiang provincial natural science foundation of China and the Grant No. Y201327415 of Zhejiang Provincial Education Department.

Received: 28 November 2013 Accepted: 9 May 2014 Published: 22 May 2014

\section{References}

1. Babbage, C: An essay towards the calculus of functions. Philos. Trans. R. Soc. Lond. Ser. A 105, 389-423 (1815)

2. Babbage, C: An essay towards the calculus of functions, II. Philos. Trans. R. Soc. Lond. Ser. A 106, $179-256$ (1816)

3. Bödewadt, UT: Zur Iteration reeller Funktionen. Math. Z. 49, 497-521 (1944)

4. Kuczma, M: On the functional equation $\varphi^{n}(x)=g(x)$. Ann. Pol. Math. 11, 161-175 (1961)

5. Isaacs, R: Iterates of fractional order. Can. J. Math. 2, 409-416 (1950)

6. Kobza, J: Iterative functional equation $x(x(t))=f(t)$ with $f(t)$ piecewise linear. J. Comput. Appl. Math. 115, 331-347 (2000)

7. Targonski, G: Progress of iteration theorem since 1981. Aequ. Math. 50, 50-72 (1995)

8. Bogatyi, S: On the nonexistence of iterative roots. Topol. Appl. 76, 97-123 (1997)

9. Kuczma, M: Functional Equations in a Single Variable. Polish Sci., Warsaw (1968)

10. Kuczma, M, Choczewski, B, Ger, R: Iterative Functional Equations. Cambridge University Press, Cambridge (1990)

11. Zhang, JZ, Yang, L: Discussion on iterative roots of piecewise monotone functions. Acta Math. Sin., 26, 398-412 (1983) (in Chinese)

12. Zhang, WN: PM functions, their characteristic intervals and iterative roots. Ann. Pol. Math. 65, 119-128 (1997)

13. Blokh, A, Coven, E, Misiurewicz, M, Nitecki, Z: Roots of continuous piecewise monotone maps of an interval. Acta Math. Univ. Comen. 60, 3-10 (1991)

14. Li, L, Yang, DL, Zhang, WN: A note on iterative roots of PM functions. J. Math. Anal. Appl. 34, 1482-1486 (2008)

15. Liu, L, Zhang, WN: Non-monotonic iterative roots extended from characteristic intervals. J. Math. Anal. Appl. 378, 359-373 (2011)

16. Liu, L, Jarczyk, W, Li, L, Zhang, WN: Iterative roots of piecewise monotonic functions of nonmonotonicity height not less than 2. Nonlinear Anal. 75, 286-303 (2012)

17. Shi, YG: Iterative roots with circuits for piecewise continuous and globally periodic maps. Topol. Appl. 159, 2721-2727 (2012) 
18. Shi, YG, Chen, L: Extension of iterative roots. Aequ. Math. (2013). doi:10.1007/s00010-013-0223-9

19. Schweizer, B, Sklar, A: All trapezoid functions are conjugate. C. R. Math. Acad. Sci. 5, 275-280 (1983)

20. Schweizer, B, Sklar, A: Continuous functions that conjugate trapezoid functions. Aequ. Math. 28, 300-304 (1985)

21. Zdun, MC: On the iteration of the hat-functions. Aequ. Math. 16, 181-182 (1977)

22. Zhang, GY: Conjugation and iterative root of a class of self-mappings on the interval (II). Chin. Ann. Math., Ser. A 4 473-478 (1992) (in Chinese)

23. Sun, TX, Xi, HJ: Iterative solutions of level-top bimodal continuous self-maps on the interval. J. Syst. Sci. Math. Sci. 21, 348-361 (2001) (in Chinese)

10.1186/1029-242X-2014-204

Cite this article as: Lin: Existence of iterative roots for the sickle-like functions. Journal of Inequalities and Applications 2014, 2014:204

Submit your manuscript to a SpringerOpen ${ }^{\odot}$ journal and benefit from:

- Convenient online submission

- Rigorous peer review

Immediate publication on acceptance

- Open access: articles freely available online

- High visibility within the field

- Retaining the copyright to your article

Submit your next manuscript at $>$ springeropen.com 